\title{
INFORMATION PATH FROM RANDOMNESS AND UNCERTAINTY TO INFORMATION, HERMODYNAMICS, AND INTELLIGENCE OF OBSERVER
}

\author{
Vladimir S. Lerner \\ Marina Del Rey, CA 90292, USA,
}

\begin{abstract}
The introduced path unites uncertainty of random process and observer information process directed to certainly. Bayesian integral functional measure of entropy-uncertainty on trajectories of Markov multidimensional process is cutting by interactive impulses. Information path functional integrates multiple hidden information contributions of the cutting process correlations in information units, cooperating in doublets-triplets, bound by free information, and enfolding the sequence of enclosing triplet structures in the information network (IN) that successively decreases the entropy and maximizes information.

The IN bound triplets release free information rising information forces attracting, ordering, structuring information units, encoding doublet-triplet logic, composing quantum, classical computation, integrating memory and cognition in intelligent observer.

\section{KEYWORDS}

Random process, Probability symmetry, Uncertain entropy functional, Interactive impulse cutoff, Virtual measurement, Minimax law, Certain information path functional, Cooperative micro-macro information dynamics, Hierarchical network, Logical computation, Objective-subjective observers threshold, Selfforming intellect
\end{abstract}

\section{INTRODUCTION}

The study focuses on integration of multiple random interactions into distinctive information processes on observer, considered independently of its origin and various physical phenomena, whose specifics are still mostly unknown. It allows us to concentrate on information process itself whose self-arising creates the information observer headed to certainty and intelligence.

Physical approach to the observer, developed in Copenhagen interpretation of quantum mechanics [13], requires an act of observation, as a physical carrier of the observer knowledge, but this role is not described in the formalism of quantum mechanics.

A. Weller has included the observer in wave function [4], while according to standard paradigm: Quantum Mechanics is Natural [5-6]. The concept of publications [7,8] states that in Quantum Bayesianism, which combines quantum theory with probability theory, 'the wave function does not exists in the world-rather it merely reflects an individual's mental state.'

We have shown (in [10-16]) and in this paper that quantum information processes (rather than Quantum Particles), resulting from Bayesian form of entropy integral measure, arise in observer at conversion of the process hidden uncertainty to the equivalent certainty-information path functional measure. 
International Journal o n Information Theory (IJIT ), Vol.3, No.4, October 2014

The paper analytically identifies an integral information path that unites uncertainty of potential observable random process and observer's certain-information processes with quantum information dynamics and informational macrodynamics overcoming their gap toward the observer intelligence. The path is formed by a sequence of axiomatic probability distributions which transfers $a$ priori probabilities to a posteriori probabilities through a stochastic multi-dimensional process whose trajectories alternates this probability's sequence over the process. Arising Bayesian entropy for these probabilities defines an uncertainty measure along the process.

The probability's transitions model an interactive random process, generated by an idealized (virtual) measurement of uncertainty, as the observable process of a potential observer. When the various virtual measurements, testing uncertainty by interactive impulses of the observable process, reveal its certain a posteriori probability, this inferring probability's test-impulse starts converting uncertainty to certainty-information. The uncertain impulse becomes a certain impulse control which extracts the maximum of the process information from each of its observed minima and initiates both the information and its observer with its internal process during the conversion path.

Multiple trial actions produce the observed experimental frequency of the measured probability of those events which actually occurred.

The minimax is dual complementary principle by which the optimal max-min extraction and minimax consumption of information establish mathematical information law whose variation equations determine the structure of the observer and functionally unify its regularities.

The impulse controls cut off the maxmin information, convert the observable external process to optimal internal information micro- and macrodynamics through integral measuring, multiple trials, verification the symmetry of the trial information, memorizing, cooperation and enfoldment in information network (IN), its logical hierarchical information structure, which provides measuring quality of information in the IN hierarchical locations and a feedback path to observations.

The IN high-level logic originates the observer's information intelligence, requesting new quality information.

These functional regularities create united self-operating information mechanism whose integral logic transforms multiple interacting uncertainties to the observer self-forming inner dynamic and geometrical structures within a limited boundary which is shaped by the IN information geometry during time-space cooperative processes and finally to physical substance, human information and cognition, which originates the intelligent observer. The operations with classical information units, cooperated from the quantum information units that IN runs, model a classical computation.

The IN different hierarchical levels unite logic of quantum micro- and macro- information processes, composing quantum and/or classical computations. The logic holds invariance of the information and physical regularities, following from the minimax information law.

The informational nature of multiple interactive processes is applying in human observations, Humanmachine communications, biological, social, economic, and other interactive systems, which the information observer collects and integrates interacting with these sources at micro- and macro levels.

\subsection{OBSERVING RANDOM UNCERTAIN PROCESS AND ITS INTEGRAL MEASURE}

Suppose a manifold of various spontaneous occurrences represents a multi-dimensional interactive random process, distributing in space-time, for example, earthquakes, instantaneous changes in stock market, or atomic explosion. Theoretically, a field of multiple distributed probabilities initiates the 
International Journal o n Information Theory (IJIT ), Vol.3, No.4, October 2014

random process with alternating a priori -a posteriori probabilities along the process trajectories, where each transfer from priori to posteriori probability distribution follows from interaction along the multi-dimensional process.

Formal model of a observed random nonstationary interactive process describes Markov diffusion process as solutions of Ito' the $n$-dimensional controlled stochastic equation:

$d \tilde{x}_{t}=a\left(t, \tilde{x}_{t}, u_{t}\right) d t+\sigma\left(t, \tilde{x}_{t}\right) d \xi_{t}, \tilde{x}_{s}=\eta, t \in[s, T]=\Delta, s \in[0, T] \subset R_{+}^{1}$

with the standard limitations [9] on drift function $a\left(t, \tilde{x}_{t}, u_{t}\right)=a^{u}\left(t, \tilde{x}_{t}\right)$, depending on control $u_{t}$, diffusion $\sigma\left(t, \tilde{x}_{t}\right)$, and Wiener process $\xi_{t}=\xi(t, \omega)$, which are defined on a probability space of the elementary random events $\omega \in \Omega$ with the variables located in $R^{n} ; \tilde{x}_{t}=\tilde{x}(t)$ is a diffusion process, as a solution of (1.1) under control $u_{t}$; $\Psi(s, t)$ is a $\sigma$-algebra created by the events $\{\tilde{x}(\tau) \in B\}$, and $P(s, \tilde{x}, t, B)$ are transition probabilities on $s \leq \tau \leq t ; P_{s, x}=P_{s, x}(A)$ are the corresponding conditional probability's distributions on an extended $\Psi(s, \infty) ; E_{s, x}[\square$ are the related mathematical expectations.Suppose control function $u_{t}$ provides transformation of an initial process $\tilde{x}_{t}$, with transition probabilities $P(s, \tilde{x}, t, B)$, to other diffusion process $\tilde{x}_{t}^{\sigma}=\int_{s}^{t} \sigma\left(v, \zeta_{v}\right) d \zeta_{v}$ as solution of (1.1) at $a\left(t, \tilde{x}_{t}, u_{t}\right)=0$ (that models an uncontrollable noise with $E\left[\tilde{x}_{t}^{a}\right]=\mathrm{O}$ ), with transitional probability's functions

$$
\tilde{P}\left(s, \tilde{x}_{t}^{\sigma}, t, B\right)=\int_{\tilde{x}(t) \in B} \exp \left\{-\varphi_{s}^{t}(\omega)\right\} P_{s, x}(d \omega),
$$

where $\varphi_{s}^{t}=\varphi_{s}^{t}(\omega)$ is an additive functional of process $\tilde{x}_{t}=\tilde{x}(t)$ [9], measured regarding $\Psi(s, t)$ at any $s \leq \tau \leq t$ with probability 1 , and $\varphi_{s}^{t}=\varphi_{s}^{\tau}+\varphi_{\tau}^{t}, E_{s, x}\left[\exp \left(-\varphi_{s}^{t}(\omega)\right)\right]<\infty$.

This transformation determines extensive distributions $\tilde{P}_{s, x}=\tilde{P}_{s, x}(A)$ on $\Psi(s, \infty)$ with density measure

$p(\omega)=\frac{\tilde{P}_{s, x}}{P_{s, x}}=\exp \left\{-\varphi_{s}^{t}(\omega)\right\}$

whose a posteriori probability distribution $\tilde{P}_{s, x} \rightarrow P_{s, x}^{p}$ and a priory probability distribution $P_{s, x} \rightarrow P_{s, x}^{a}$ alternates along the process trajectories.

The additive functional determines the solutions of (1.1) in form [9]:

$$
\begin{aligned}
& \varphi_{s}^{T}=1 / 2 \int_{s}^{T} a^{u}\left(t, \tilde{x}_{t}\right)^{T}\left(2 b\left(t, \tilde{x}_{t}\right)\right)^{-1} a^{u}\left(t, \tilde{x}_{t}\right) d t+\int_{s}^{T}\left(\sigma\left(t, \tilde{x}_{t}\right)^{-1} a^{u}\left(t, \tilde{x}_{t}\right) d \xi(t),\right. \\
& 2 b(t, \tilde{x})=\sigma(t, \tilde{x}) \sigma^{T}(t, \tilde{x})>0
\end{aligned}
$$

Using definition the entropy for these processes as math expectation of (1.2) taken for each posteriori probability along the process' trajectories: 
International Journal o n Information Theory (IJIT ), Vol.3, No.4, October 2014

$\Delta S\left[\tilde{x}_{t}\right]=-E_{s, x}[\ln p(\omega)]=\int_{\tilde{x}(t) \in B}-\ln [p(\omega)] P_{s, x}^{p}(d \omega)$

at $E\left[\int_{s}^{T}\left(\sigma\left(t, \tilde{x}_{t}\right)^{-1} a^{u}\left(t, \tilde{x}_{t}\right) d \xi(t)\right]=0\right.$, we get entropy functional (EF) on the process trajectories, expressed through regular $a^{u}\left(t, \tilde{x}_{t}\right)$ and stochastic components $b\left(t, \tilde{x}_{t}\right)$ of diffusion process $\tilde{x}_{t}$ :

$$
\left.\Delta S\left[\tilde{x}_{t}\right]\right|_{s} ^{T}=1 / 2 E_{s, x}\left\{\int_{s}^{T} a^{u}\left(t, \tilde{x}_{t}\right)^{T}\left(2 b\left(t, \tilde{x}_{t}\right)\right)^{-1} a^{u}\left(t, \tilde{x}_{t}\right) d t\right\}
$$

The EF is an information indicator of distinction between processes $\tilde{x}_{t}$ and $\tilde{x}_{t}^{\sigma}$ by these processes' measures; it measures quantity of information of process $\tilde{x}_{t}$ regarding process $\tilde{x}_{t}^{\sigma}$ brought by all hidden randomness through each infinitely small instant as the process' differential $d \omega$.

For the process' equivalent measures, this quantity is zero, and it is a positive for the process' nonequivalent measures.

Quantity of information (1.4) is equivalent of Kullback-Leibler's divergence (KL) for continiuos random variables:

$D_{K L}\left(P_{x, s}^{a} \| P_{x, s}^{p}\right)=\int_{X} \ln \frac{d P^{a}}{d P^{p}} d P^{p}=E_{x}\left[\ln \frac{P_{x, s}^{a}(d \omega)}{P_{x, s}^{p}(d \omega)}\right]=E_{x}[\ln p(\omega)]$,

where $\frac{d P^{a}}{d P^{p}}$ is distance's measure between the associated entropies.

The KL measure is connected to both Shannon's conditional information and Bayesian inference of testing a priory hypothesis (probability distribution) by a posteriori observation's probability distribution.

The observer EF implements the Bayesian inference by intervening in the following process location as a causal probability.

The process' multiple state connections, changing a nearest probability transformation, automatically integrate the transformation (interaction) of these alternating probabilities along the process trajectory. Some spread of these interactions (that might differ in the dimensions) we define as an observable (virtual) process of a potential observer.

In a set of process' events occurrences, each occurrence has probability 1, which excludes others with probability 0. According to Kolmogorov's 0-1 law [17], these experimental multiple frequencies enables predicting axiomatic Kolmogorov probability $P_{s, x}$, if the experiment manifold satisfies condition of symmetry of equal probable events [18].

Thus, the observable process' multiple interactions in a form of impulses hold virtual probing Yes-No (1-0) actions, whose multiple frequencies enable generating both a priori and a posteriori probabilities, 
International Journal o n Information Theory (IJIT ), Vol.3, No.4, October 2014

their relational probabilities and the uncertainty integral measure (EF) for each observable process dimension.

Such interactions could be both a natural Markov process and artificially generated probs during the observable process, while the interactive connections hold integral measure of hidden uncertainties (entropy) along the process.

\section{THE MINIMAX CUTOFF AT EACH OBSERVATION. ESTIMATION OF PROBABILITIES AND THE FUNCTIONAL MEASURES}

The observation of uncertainty measure requires an infinitesimal small portion of each process, holding an impulse form, which concurrently changes the process' probabilities, starting with measuring uncertainty and ending with reaching certainty.

In the observable multi-dimensional Markov process, each probing a priori probability turns to the following a posteriori probability, cutting off uncertainty and converting it to certainty, which the certain impulses encode as information, and add the information to its integral measure.

Let define the control on space $K C(\Delta, U)$ for piece-wise continuous step functions $u_{t}$ at $t \in \Delta$ :

$u_{-} \stackrel{\operatorname{def}}{=} \lim _{t \rightarrow \tau_{k}-o} u\left(t, \tilde{x}_{\tau_{k}}\right), u_{+} \stackrel{\operatorname{def}}{=} \lim _{t \rightarrow \tau_{k}+o} u\left(t, \tilde{x}_{\tau_{k}}\right)$,

which are differentiable on the set

$$
\Delta^{o}=\Delta \backslash\left\{\tau_{k}\right\}_{k=1}^{m}, k=1, \ldots, m
$$

and applied on diffusion process $\tilde{x}_{t}$ from moment $\tau_{k-o}$ to $\tau_{k}$, and then from moment $\tau_{k}$ to $\tau_{k+o}$, implementing the process' transformations $\tilde{x}_{t}\left(\tau_{k-o}\right) \rightarrow \tilde{x}_{t}^{\sigma}\left(\tau_{k}\right) \rightarrow \tilde{x}_{t}\left(\tau_{k+o}\right)$.

At a vicinity of moment $\tau_{k}$, between the jump of control $u_{-}$and the jump of control $u_{+}$, we consider a control impulse:

$\delta u_{ \pm}\left(\tau_{k}\right)=u_{-}\left(\tau_{k-o}\right)+u_{+}\left(\tau_{k+o}\right)$.

The following Proposition evaluates the entropy functional contributions at such transformations.

Entropy functional $(1.4,1.5)$ at the switching moments $t=\tau_{k}$ of control (2.2) takes the values

$\Delta S\left[\tilde{x}_{t}\left(\delta u_{ \pm}\left(\tau_{k}\right)\right]=1 / 2\right.$,

and at locality of $t=\tau_{k}$ : at $\tau_{k-o} \rightarrow \tau_{k}$ and $\tau_{k} \rightarrow \tau_{k+o}$, produced by each of the impulse control's step functions in (2.2), is estimated accordingly by

$\Delta S\left[\tilde{x}_{t}\left(u_{-}\left(\tau_{k}\right)\right]=1 / 4, u_{-}=u_{-}\left(\tau_{k}\right), \tau_{k-o} \rightarrow \tau_{k}\right.$,

$\Delta S\left[\tilde{x}_{t}\left(u_{+}\left(\tau_{k}\right)\right]=1 / 4, u_{+}=u_{+}\left(\tau_{k}\right), \tau_{k} \rightarrow \tau_{k+o}\right.$. 
International Journal o n Information Theory (IJIT ), Vol.3, No.4, October 2014

Proof. The jump of the control function $u_{-}$in (2.1) from a moment $\tau_{k-o}$ to $\tau_{k}$, acting on the diffusion process, might cut off this process after moment $\tau_{k-o}$. The cut off diffusion process has the same drift vector and the diffusion matrix as the initial diffusion process.

The additive functional for this cut off has the form:

$\varphi_{s}^{t-}=\left\{\begin{array}{l}0, t \leq \tau_{k-o} \\ \infty, t>\tau_{k}\end{array}\right.$

The jump of the control function $u_{+}$(2.1) from $\tau_{k}$ to $\tau_{k+o}$ might cut off the diffusion process after moment $\tau_{k}$ with the related additive functional

$\varphi_{s}^{t+}=\left\{\begin{array}{l}\infty, t>\tau_{k} \\ 0, t \leq \tau_{k+o}\end{array}\right.$.

For the control impulse (2.2), the additive functional at a vicinity of $t=\tau_{k}$ acquires the form of an impulse function

$\varphi_{s}^{t-}+\varphi_{s}^{t+}=\delta \varphi_{s}^{\mp}$

which summarizes (2.4a) and (2.4b).

Entropy functional $(1.4,1.5)$, following from $(2.4 \mathrm{a}, \mathrm{b})$, takes the values

$$
\begin{gathered}
\Delta S\left[\tilde{x}_{t}\left(u_{-}\left(t \leq \tau_{k-o} ; t>\tau_{k}\right)\right)\right]=E\left[\varphi_{s}^{t-}\right]=\left\{\begin{array}{l}
0, t \leq \tau_{k-o}, \\
\infty, t>\tau_{k}
\end{array},\right. \\
\Delta S\left[\tilde{x}_{t}\left(u_{+}\left(t>\tau_{k-o} ; t \leq \tau_{k+o}\right)\right)\right]=E\left[\varphi_{s}^{t+}\right]=\left\{\begin{array}{l}
\infty, t>\tau_{k-o} \\
0, t \leq \tau_{k+o}
\end{array},\right.
\end{gathered}
$$

changing from 0 to $\infty$ and back from $\infty$ to 0 and acquiring an absolute maximum at $t>\tau_{k}$, between $\tau_{k-o}$ and $\tau_{k+o}$. The multiplicative functionals [9] related to (2.4 a,b), are:

$p_{s}^{t-}=\left\{\begin{array}{l}0, t \leq \tau_{k-o} \\ 1, t>\tau_{k}\end{array}, \quad p_{s}^{t+}=\left\{\begin{array}{l}1, t>\tau_{k} \\ 0, t \leq \tau_{k+o}\end{array}\right.\right.$.

Impulse control (2.2) provides an impulse probability density in the form of multiplicative functional

$$
\delta p_{s}^{\mp}=p_{s}^{t-} p_{s}^{t+}
$$

where $\delta p_{s}^{\mp}$ holds $\delta\left[\tau_{k}\right]$-function, which determines the process' transitional probabilities with $\tilde{P}_{s, x}(d \omega)=0$ at $t \leq \tau_{k-o}, t \leq \tau_{k+o}$ and $\tilde{P}_{s, x}(d \omega)=P_{s, x}(d \omega)$ at $t>\tau_{k}$.

For the cutoff diffusion process, the transitional probability (at $t \leq \tau_{k-o}$ and $t \leq \tau_{k+o}$ ) turns to zero, and states $\tilde{x}\left(\tau_{k}-o\right), \tilde{x}\left(\tau_{k}+o\right)$ become independent, while their mutual time correlations are dissolved: 
International Journal o n Information Theory (IJIT ), Vol.3, No.4, October 2014

$$
r_{\tau_{k}-o, \tau_{k}+o}=E\left[\tilde{x}\left(\tau_{k}-o\right), \tilde{x}\left(\tau_{k}+o\right)\right] \rightarrow 0
$$

Entropy increment $\Delta S\left[\tilde{x}_{t}\left(\delta u_{ \pm}\left(\tau_{k}\right)\right)\right]$ for additive functional $\delta \varphi_{s}^{\mp}(2.5)$, which is produced within, or at a border of the control impulse (2.2), is defined by the equality

$$
E\left[\varphi_{s}^{t-}+\varphi_{s}^{t+}\right]=E\left[\delta \varphi_{s}^{\mp}\right]=\int_{\tau_{k-o}}^{\tau_{k+o}} \delta \varphi_{s}^{\mp}(\omega) P_{\delta}(d \omega),
$$

where $P_{\delta}(d \omega)$ is a probability evaluation of the impulse $\delta \varphi_{s}^{\mp}$.

Taking integral of symmetric $\delta$-function $\delta \varphi_{s}^{\mp}$ between above time intervals, the border gets

$$
E\left[\delta \varphi_{s}^{\mp}\right]=1 / 2 P_{\delta}\left(\tau_{k}\right) \text { at } \tau_{k}=\tau_{k-o} \text {, or } \tau_{k}=\tau_{k+o}
$$

The impulse, produced by deterministic controls (2.2) for each random process dimension, is a random with probability at $\tau_{k}$-locality

$$
P_{\delta c}\left(\tau_{k}\right)=1, k=1, \ldots, m
$$

This probability holds a jump-diffusion transition Markovian probability, which is conserved during the jump.

From (2.10)-(2.12) we get estimation of the entropy functional's increment, when the impulse control (2.2) is applied (at $t=\tau_{k}$ for each $k$ ), in the form

$$
\Delta S\left[\tilde{x}_{t}\left(\delta u_{ \pm}\left(\tau_{k}\right)\right)\right]=E\left[\delta \varphi_{s}^{\mp}\right]=1 / 2,
$$

which proves (2.3).

Since that, each of symmetrical information contributions (2.6a,b) at a vicinity of $t=\tau_{k}$ estimate

$$
\begin{aligned}
& \Delta S\left[\tilde{x}_{t}\left(u_{-}\left(t \leq \tau_{k-o} ; t>\tau_{k}\right)\right)\right]=1 / 4, u_{-}=u_{-}\left(\tau_{k}\right), \tau_{k-o} \rightarrow \tau_{k}, \\
& \Delta S\left[\tilde{x}_{t}\left(u_{+}\left(t>\tau_{k-o} ; t \leq \tau_{k+o}\right)\right)\right]=1 / 4, u_{+}=u_{+}\left(\tau_{k}\right), \tau_{k} \rightarrow \tau_{k+o},
\end{aligned}
$$

which proves $(2.3 \mathrm{a}, \mathrm{b}) \cdot \bullet$

Entropy functional (EF) (1.5), defined through Radon-Nikodym's probability density measure (1.2), holds all properties of the considered cutoff controllable process, where both $P_{s, x}$ and $\tilde{P}_{s, x}$ are defined. That includes abilities for measuring $\delta$-cutoff information and extracting a hidden process information not measured by known information measures.

Hence, information measures the cutoff interaction which had bound and hidden by the interaction's uncertainty measure. According to the definition of entropy functional (1.4), it is measured in natural ln, where each of its Nat equals $\log _{2} e \cong 1.44$ bits .

Thus, measure $(1.4,1.5)$ is not using Shannon entropy measure.

From $(2.6 a, b)$ and $(1.4,1.2)$ follow Corollaries: 


\section{International Journal o n Information Theory (IJIT ), Vol.3, No.4, October 2014}

a. Step-wise control function $u_{-}=u_{-}\left(\tau_{k}\right)$, implementing transformation $\tilde{x}_{t}\left(\tau_{k-o}\right) \rightarrow \tilde{x}_{t}^{\sigma}\left(\tau_{k}\right)$, converts the entropy functional from its minimum at $t \leq \tau_{k-o}$ to the maximum at $\tau_{k-o} \rightarrow \tau_{k}$;

b. Step-wise control function $u_{+}=u_{+}\left(\tau_{k}\right)$, implementing transformation $\tilde{x}_{t}^{\sigma}\left(\tau_{k}\right) \rightarrow \tilde{x}_{t}\left(\tau_{k+o}\right)$, converts the entropy functional from its maximum at $t>\tau_{k}$ to the minimum at $\tau_{k} \rightarrow \tau_{k+o}$;

c. Impulse control function $\delta u_{\tau_{k}}^{\mp}$, implementing transformations $\tilde{x}_{t}\left(\tau_{k-o}\right) \rightarrow \tilde{x}_{t}^{\sigma}\left(\tau_{k}\right) \rightarrow \tilde{x}_{t}\left(\tau_{k+o}\right)$, switches the entropy functional from its minimum to maximum and back from maximum to minimum, while the absolute maximum of the entropy functional at a vicinity of $t=\tau_{k}$ allows the impulse control to deliver maximal amount of information (2.13) from these transformations, holding principle of extracting maxmin- minmax of the EF measure;

d. Dissolving the correlation between the process' cutoff points (2.9) leads to losing the functional connections at these discrete points, which evaluate the Feller kernel measure of the Markov diffusion process [9].

e. The relation of that measure to additive functional in form (1.3) allows evaluating the kernel's information by the entropy functional (1.5).

f. The jump action (2.1) on Markov process, associated with "killing its drift", selects the Feller measure of the kernel, while the functional cutoff provides entropy-information measure of the Feller kernel, and it is a source of a kernel information, estimated by (2.13).

In a multi-dimensional diffusion process, each of the stepwise control, acting on the process' all dimensions, sequentially stops and starts the process, evaluating the multiple functional information. The dissolved element of the functional's correlation matrix at these moments provides independence of the cutting off fractions, leading to orthogonality of the correlation matrix for these cutoff fractions. g. A multi-dimensional delta-distribution is the minimax optimal to hold the dissolving interacting correlations, which best approaches the Tracy-Widom distribution for complex interactions.

Let us consider a sum of increments (2.13) under impulse control $\delta u\left(\tau_{k}\right)$, cutting process $x_{t}$ at moments $\tau_{k}, k=1,2, \ldots, m$, along the process' trajectory on intervals $s>\tau_{1}>t_{1}>\tau_{2}>t_{2}, \ldots, t_{m-1}>\tau_{m}>t_{m}=T$.

Applying additive principle for the process' information functional, measured at the moments of dissolving the correlation, which provides maximal cut off information, we get sum

$S_{m}=\sum_{k=1}^{m} \Delta S_{k}\left[\tilde{x}_{t}\left(\delta u\left(\tau_{k}\right)\right)\right]=\Delta S_{1}\left[\tilde{x}_{t}\left(\delta u\left(\tau_{1}\right)\right)\right]+\Delta S_{2}\left[\tilde{x}_{t}\left(\delta u\left(\tau_{2}\right)\right)\right] \mid, \ldots,+\Delta S_{m}\left[\tilde{x}_{t}\left(\delta u\left(\tau_{m}\right)\right)\right]$.

Impulses $\delta u\left(\tau_{k}\right)$ implement the transitional transformations (1.2), initiating the Feller kernels along the process and extracting total kernel information for $n$-dimensional process with $m$ cutoff. This maximal sum measures the interstates information connections held by the process along the trajectories during its time interval $(T-s)$. It measures information hidden by the process correlating states, which is not covered by traditional Shannon entropy measure.

This sum of extracted information approaches theoretical measure (1.4) at

$\left.S_{m}\right|_{s} ^{T} \underset{m \rightarrow \infty}{\longrightarrow} S\left[\tilde{x}_{t} / \varsigma_{t}\right]_{s}^{T}$,

if all local time intervals $t_{1}-s=o_{1}, t_{2}-t_{1}=o_{2}, \ldots, t_{m}-t_{m-1}=o_{m}$, at $t_{m}=T$ satisfy condition

$(T-s)=\lim _{m \rightarrow \infty} \sum_{t=s, m}^{t=T} o_{m}(t)$. 
International Journal o n Information Theory (IJIT ), Vol.3, No.4, October 2014

Realization of (2.15) requires applying the impulse controls at each instant $(\tilde{x}, s),(\tilde{x}, s+o(s))$ of the conditional mathematical expectation (1.4) along the process trajectories.

However for any finite number $m$ (of instant $(\tilde{x}, s),(\tilde{x}, s+o(s))$ ) the integral process information (1.5) cannot be exactly composed from the information, measured for the process' fractions.

Indeed, sum $\left.S_{m o}\right|_{s} ^{T}$ of additive fractions of (2.4) on the finite time intervals: $s, t_{1} ; t_{1}+o_{1}, t_{2} ; . ., t_{m-1}, t_{m-1}+o_{m} ; t_{m}=T$ :

$\left.\left.S_{m o}\right|_{s} ^{T}=\Delta S_{1 o}\left[\tilde{x}_{t} / \varsigma_{t}\right] l_{s}^{t_{1}}+\Delta S_{2 o}\left[\tilde{x}_{t} / \varsigma_{t}\right]\right]_{t_{1}+o_{1}}^{t_{2}}, \ldots,+\left.\Delta S_{m o}\left[\tilde{x}_{t} / \zeta_{t}\right]\right|_{t_{m-1}+o_{m}} ^{t_{m}}$

is less than $S\left[\tilde{x}_{t} / \varsigma_{t}\right]_{s}^{T}$, which is defined through the additive functional (1.5).

As a result, the additive principle for a process' information, measured by the $\mathrm{EF}$, is violated:

$\left.S_{m}\right|_{s} ^{T}<S\left[\tilde{x}_{t} / \varsigma_{t}\right]_{s}^{T}$.

If each $k$-cutoff "kills" its process dimension after moment $\tau_{k+o}$, then $m=n$, and (2.15) requires infinite process dimensions. A finite dimensional model approximates finite number of probing impulses checking the observing frequencies. For any of these conditions, the EF measure, taken along the process trajectories during time interval $(T-s)$, limits maximum of total process information, extracting its hidden cutoff information (during the same time), and brings more information than Shannon traditional information measure for multiple states of the process. Therefore, maximum of the process cutoff information, extracting its total integrated hidden information, approaches the EF information measure. Since the maxmin probes automatically minimize current entropy measure, its actual measurement is not necessary.

Writing sum (2.14) in matrix form for the multiple process' independent components considered during $(T-s)$, we can represent it as the matrix trace:

$\left.S_{m o}\right|_{s} ^{T}=\operatorname{Tr}\left[\Delta S_{k}\left[\tilde{x}_{t}\left(\delta u\left(\tau_{k}\right)\right)\right], k=1, \ldots . . n, m=n\right.$,

which relates (2.28) to Von Neumann entropy for quantum ensemble [3].

Since entropy (1.4), (2.18) measures information of the process' hidden variable, while each cutoff initiates the quantum dynamic Schrödinger's bridge, whose information measures Von Neumann's entropy of the bridges [13] for all process' dimensions, both of these measures (2.18) get connected by the impulse control's cutoff.

Relation (2.18) satisfies such impulse control that kills each dimension by stopping its process at the cutoff [12]. For $n \rightarrow \infty$, the Von Neumann entropy (2.18) approaches the information measure (2.16), which, being equal to entropy functional $(\mathrm{EF})(1.5)$, determines information path functional (IPF) as integral certainty measure of EF.

The IPF is defined on a dynamic process as the IPF extremal, which is the dynamic equivalent of initial random process. The IPF is dynamic counterpart of EF, which is defined on irreversible random process, while the IPF dynamic process is reversible and certain.

\section{INFORMATION MECHANISM OF ROTATION AND ORDERING COLLECTED INFORMATION}

The mechanism forms triplets and joint them in observer information structure while ordering information from the cutoff observations and implementing the minimax principle. 


\section{International Journal o n Information Theory (IJIT ), Vol.3, No.4, October 2014}

Let us have distributed in space interactive $n$-dimensional random process $\tilde{x}_{t}^{*}=\tilde{x}\left(t^{*}, l^{*}\left(t^{*}\right)\right)$, where space parameter $l^{*}\left(t^{*}\right)$ is non-random function of time course $t^{*}$.

The process is described by solutions of Ito stochastic differential equation with drift function $a^{u}\left(t^{*}, l^{*}\left(t^{*}\right)\right)$ and diffusion $\sigma\left(t^{*}, l^{*}\left(t^{*}\right)\right)$ depending on the space parameter, while each interaction depicts delta function $\delta S_{\tilde{x} u}=\mathrm{u}_{o i} \delta\left(t^{*}, l^{*}\left(t^{*}\right)\right)$ at each $\left(t^{*}, l^{*}\left(t^{*}\right)\right)$ with delta impulse $\delta\left(t^{*}, l^{*}\left(t^{*}\right)\right)$ along trajectory of this multi-dimensional random process.

Such interaction holds hidden uncertainty $\mathrm{u}_{o i}$, covering the process' bound interstates connections, which measures entropy functional $S_{a p}$ along the random process; $\mathrm{u}_{o i}$ and $S_{a p}$ might be converted to observer information $\mathbf{a}_{i}$ and information path functional $I_{a p}$ accordingly.

Let us consider classical transformation of coordinate system with vector $\vec{l}^{*}\left(t^{*}\right)=\left(l_{x}^{*}, l_{y}^{*}, l_{z}^{*}\right)$ at moment $t^{*}$, defined in moving coordinate system, with vector $\vec{l}(t)=\left(l_{x}, l_{y}, l_{z}\right)$ in immobile coordinate system at moment $t^{*}+\delta t^{*}=t$ :

$\vec{l}^{*}\left(t^{*}\right)=\dot{\bar{A}}\left(t^{*}\right)\left[\vec{l}+\vec{L}\left(t^{*}\right)\right]$,

where $\bar{A}\left(t^{*}\right)$ is orthogonal matrix of rotation for mobile coordinate system, $\vec{L}\left(t^{*}\right)$ is a vector of shift from the origin of this coordinate system relatively to vector $\vec{l}$. Assuming observing process $\tilde{x}_{t}=\tilde{x}(t, \vec{l}(t))$ locates in immobile coordinate system and observable process $\tilde{x}_{t}^{*}$ locates in the moving coordinate system, the relative motion the coordinate system in holds the form

$\vec{l}(t)=\bar{A}(t)^{-1}\left[\vec{l}^{*}-\vec{L}\right]$,

where $\vec{l}$ is coordinate vector of the same space point in immobile coordinate system, $\bar{A}$ is orthogonal matrix of rotation in immobile coordinate system, $\vec{L}$ is vector of shift in this coordinate system.

Then a single-parametrical family of transformation of parameter $t^{*}+\delta t^{*}=t$, which takes into account time-shift of these coordinate systems, approximates relation [10]:

$\partial \vec{l} / \partial t \cong \dot{\bar{A}}(t) \bar{A}(t)^{-1}[\vec{l}-\vec{L}]+\dot{\vec{L}}, \quad \ddot{\bar{L}}=\dot{\bar{A}}(t) \bar{A}(t)^{-1} \dot{\bar{L}}$.

A skew-symmetrical velocity tensor of rotation $W=\left(W_{i o}^{+}, W_{i o}^{-}\right)$, which has a group representation with inverse rotating velocities for each $i$ impulse $W_{i o}=\left(W_{i+}, W_{i-}\right)$, follows from matrix Eqs:

$$
\dot{\bar{A}}(t) \bar{A}(t)^{-1}=W \exp (W t) \exp (-W t)=W
$$

According to Noether's theorem, conversion of the external process' uncertainty (EF) functional to the observer's process' certainty-information path functional (IPF) is invariant under this transformation, satisfying the variation principle, where for the space shift $\delta \vec{l}$, which can be observed in an immobile system, the invariant conditions must be preserved during a time $t=\delta t+t_{o}$.

The VP Lagrange-Hamilton Eqs prognosis the observer process' conjugated extremal trajectories of distributed dynamics process $x_{t}=x(t, l(t))$ by solutions of two differential Eqs:

$$
\begin{aligned}
& \partial x_{i} / \partial t+1 / 2 \sum_{k=1}^{3} \partial x_{i} / \partial l_{k}\left\{\left[W_{i+}\left(l_{k}(t)-L_{k}(t)\right]+\dot{\vec{L}}_{k}(t)\right\}=0,\right. \\
& \partial x_{i} / \partial t+1 / 2 \sum_{k=1}^{3} \partial x_{i} / \partial l_{k}\left\{\left[W_{i-}\left(l_{k}(t)-L_{k}(t)\right]+\dot{\vec{L}}_{k}(t)\right\}=0,\right.
\end{aligned}
$$


International Journal o n Information Theory (IJIT ), Vol.3, No.4, October 2014

where $W_{i+}, W_{i-}$ are the opposite velocities components of tensor (3.3a), applied to the cutting $k$-space coordinates of each $i$-process' trajectories for all $n$ dimensions of the process, at different initial conditions for $k, i$. These Egs. include gradient form, determined in the coordinate systems, connected by the transformation (3.1):

$\frac{\partial x_{i}}{\partial l_{j}}=\sum_{k=1}^{3}\left(\frac{\partial x_{i}}{\partial l_{k}^{o}}\right) \bar{a}_{k j},\left(\bar{a}_{k j}\right)=\bar{A}^{-1} ; k, j=1,2,3 ; \operatorname{gradx}_{i}=\bar{A} \operatorname{grad}^{o} x_{i}$,

with primary gradient $\operatorname{grad}^{o} x_{i}$, and the extremal equations of diffusion process:

$\frac{\partial x_{m}}{\partial t}=a_{m}(t, x, u), a_{m}=A\left(x+v(\tau, \vec{l}), A=A^{T}, m=1, \ldots, n\right.$,

$1 / 2 \sigma \sigma^{T}=b, b=b(t, x), b, A: \Delta \times R^{3} \rightarrow \angle\left(R^{n}\right)$,

holding control function $u=A v(\tau, \vec{l})$ of observer inner control $v(\tau, \bar{l})$.

A cutting component of observable process' diffusion operator $\sigma\left(t^{*}, l^{*}\left(t^{*}\right)\right)=\left|\sigma_{i i}\right|$ identifies eigenvalue $\alpha_{i}$ of matrix $A$ (which also defines space Eqs (3.1-3.5)):

$\sigma_{i i}^{-1} \frac{\partial \sigma_{i i}}{\partial t}=\alpha_{i}$

The diagonalized model of the equation of extremals and its Lagrangian hold forms:

$\frac{\partial x_{i}^{2}}{\partial t^{2}}=\sigma_{i i}^{-1} \frac{\partial \sigma_{i i}}{\partial t} \frac{\partial x_{i}}{\partial t}, i=1, \ldots, n, L=1 / 2 \sum_{i=1}^{n} \sigma_{i i}^{-1}\left(\frac{\partial x_{i}}{\partial t}\right)^{2}$.

Lagrangian and Hamiltonian of variation principle (VP) for the multiple entropy impulses have distinct peculiarities at observer points $(t, l(t))$ of time-space distributed random process $\tilde{x}(t, l(t))$, while the EF extremals on the trajectories define functions drift and diffusion of Markov diffusion process (3.5-3.6).

\section{INFORMATION MICROPROCESSES INITIATED BY THE CUTOFF}

The opposite rotation of the conjugated entropy fractions superimposes their movement in the correlated movement, which minimizing each cutoff entropy by entangling the rotating entropy fractions in a microprocess. Thus, reaching the entanglement brings the observer's cutoff maxim-min entropy with the inverse rotating velocities to a second minimize of the maximal entropy, corresponding symmetry of the probabilities, whose repeating does not bring more entropy and limits the trajectories distinction [16].

Incursion of probing actions near and into the entanglement, prior to verification of the symmetry, does not provide measuring and memorizing entropy, being physically unperformed.

When the various virtual measurements, testing uncertainty by interactive impulses along the observable process, reveal its certain a posteriori probability, this inferring probability's test-impulse starts converting uncertainty to certainty-information.

The initial conditions of the starting dynamic process determine a boundary of the cutoff impulse, which absorbs the cutting random ensemble of observable process' states $\tilde{x}_{t}\left(\tau_{*}\right), \tilde{x}_{t}\left(\tau^{*}\right)$.

The cutoff, applied to an observable process, chooses a pair of the random ensemble states $\tilde{x}_{t}\left(\tau_{k-o}\right)=\tilde{x}_{t \max }\left(\tau_{*}\right), \tilde{x}_{t}\left(\tau_{k+o}\right)=\tilde{x}_{t \min }\left(\tau_{*}\right)$ having maximal entropy of their distinction, while $\tilde{x}_{t}\left(\tau_{*}\right)$ is transforming to $\tilde{x}_{t}\left(\tau^{*}\right)$ through the impulse. Until real cutoff, these states does not to belong to information observer, Eqs. (3.4a-c) prognosis the observer dynamics under the probing actions, which 
International Journal o n Information Theory (IJIT ), Vol.3, No.4, October 2014

potentially cutting each random micro-ensemble. At transformation of last uncertain a priori impulse to next certain a posteriori impulse, the ensemble of cutting states $\tilde{x}_{t}\left(\tau_{k-o}^{*}\right), \tilde{x}_{t}\left(\tau_{k+o}^{*}\right)$ terminate (kill) when it's transferring to initial conditions of the conversion dynamic process.

two opposite step-functions $U^{+}\left[\tilde{x}_{t \max }\left(\tau^{*}\right)\right]$ and $U^{-}\left[\tilde{x}_{t \min }\left(\tau^{*}\right)\right]$, which carrying these states, crosses the border, transforming $\tilde{x}_{t \max }\left(\tau_{*}\right)$ to $\tilde{x}_{t \text { min }}\left(\tau_{*}\right)$ with the rate of diffusion operator $\alpha_{i 2 \tau}$ (or additive functional, Sec.2) of the cutting diffusion process.

Relation for impulse cut follows from (3.5-3.6):

$U^{+}\left[\tilde{x}_{t \max }\left(\tau^{*}\right)\right]=\alpha_{i o} v_{i}^{+}, U^{-}\left[\tilde{x}_{t \min }\left(\tau^{*}\right)\right]=\alpha_{i o} v_{i}^{-}, \alpha_{i o}=1 / 2 \alpha_{i \tau}$,

if each of these actions cuts a half of the border.

In real cutoff, the boundary defines the step-up control function

$\tilde{u}^{p}\left(\tilde{x}_{t}\left(\tau_{k-o}^{*}\right), \tilde{x}_{t}\left(\tau_{k+o}^{*}\right)\right) \rightarrow \tilde{u}_{+}\left(x_{t-}\left(\tau_{k}\right), x_{t+}\left(\tau_{k}\right)\right),\left(\tau_{k-o}^{*}, \tau_{k+o}^{*}\right) \rightarrow \tau_{k}$, which, while transferring a

priori impulse to a posteriori impulse, transits the border, absorbing $\tilde{x}_{t}\left(\tau_{k-o}^{*}\right), \tilde{x}_{t}\left(\tau_{k+o}^{*}\right)$, whose cut

provides two simultaneous opposite states (with the probability $\sim 1 / 2): \tilde{x}_{t \max }\left(\tau^{*}\right)$ corresponding to

$2 \tilde{x}_{t \max }\left(\tau^{*}\right)$, and $\tilde{x}_{t \text { min }}\left(\tau^{*}\right)$ accordingly to $2 \tilde{x}_{t \text { min }}\left(\tau^{*}\right)$.

The conversion process transits with real rate of diffusion operator $\alpha_{i \tau}$ up to the moment $\tau_{k}$ when control changes sign of the states, giving start to two dynamic processes with initial conditions:

$x_{t+}\left(\tau_{k}\right)=-2 \tilde{x}_{t \max }(\tau+o), x_{t-}\left(\tau_{k}\right)=-2 \tilde{x}_{t \min }(\tau+o)$,

where

$x_{t+}\left(\tau_{k}\right)=-x_{t}\left(\tau_{k}\right)($ at $\max =-\min )$.

During time shift $t^{*}+\delta t^{*}=t$ from observable process to observation, the probing actions do not provide real rotation; it can be just virtual rotations in a time-space of the observable process with related probabilities and uncertain measure.

At the last control action, the rotating a priori impulse with elevated a priori probability, which integrate entropy of previous impulses, is transferring to rotating a posteriori certain impulse.

The step-up start is associated with acting the observer inner controls

$-2 \tilde{x}_{t \max }(\tau+o)=v^{+}$and $-2 \tilde{x}_{t \max }(\tau+o)=v^{-}$

which connect with actual control $u$ through the border diffusion $\alpha_{i \tau}$ of the cutting diffusion process. Relations $(4.2,4.3)$ follow from synthesis of the optimal controls [11] defining extermals Eqs $(3.4 \mathrm{a}, \mathrm{b})$. Transferring maximal cutting fraction $\mathrm{u}_{o i}$ of entropy functional (1.5) through the border interval $\delta_{1 i}$ (with $\alpha_{i \tau}$ ), at $\delta_{1 i} \alpha_{i \tau}=\mathrm{u}_{o i}$, corresponds to transferring time interval $\delta t^{*}$ from observable process to observer time interval $\delta t$ at $\delta t^{*} \rightarrow \delta t$. Connection $\delta t$ and $\delta \bar{l}$ in (3.3) leads to $\alpha_{i \tau} \delta t=\left(\alpha_{i \tau} \delta \vec{l}_{k}\right)^{*}\left\{\left[\left(W_{i+}\left(\vec{l}_{k}(t)-\vec{L}_{k}(t)\right)+\dot{\vec{L}}_{k}(t)\right]^{-1}+\left(\alpha_{i \tau} \delta \vec{l}_{k}\right)^{*}\left[\left(W_{i-}\left(\vec{l}_{k}(t)-\vec{L}_{k}(t)\right)+\dot{\vec{L}}_{k}(t)\right]^{-1}\right\}\right.\right.$,

(4.4) where the projection of eigenvalues (4.1) on increment of space vector $\delta \vec{l}=\delta\left(\vec{l}_{x}, \vec{l}_{y}, \vec{l}_{z}\right)$ determines $\alpha_{i \tau} \delta \vec{l}$ with its orthogonal components, while (4.4) holds scalar product of the vectors 


\section{International Journal o n Information Theory (IJIT ), Vol.3, No.4, October 2014}

sum. Multiple impulse projections maintain manifold of eigenvectors of matrix $\vec{A}=\left|\vec{\lambda}_{i}\right|, i=1, . . n$, , when each cutoff with three-dimensional $\delta \vec{l}_{\tau}$ determines three dimensional eigenvector

$\vec{a}_{i \tau}=\left|\vec{\lambda}_{i \tau}\right|, i=1,2,3, \vec{\lambda}_{i \tau}=\alpha_{i o} \delta \vec{l}_{k}$

Thus, each eigenvector is identified by related component of the diffusion operator from (3.6a).

During each $\delta t$, the observer eigenvector, according to (4.4), is under rotation with above velocities, which holds rotating eigenvector $\vec{a}_{i \tau}^{w}=\left(\vec{a}_{i \tau}^{w+}, \vec{a}_{i \tau}^{w-}\right)$ with the conjugated components:

$$
\vec{a}_{i \tau}\left\{\left[W_{i+}^{*}\left(l_{k}(t)-L_{k}(t)\right]+\dot{\vec{L}}_{k}(t)\right\}^{-1}=\vec{a}_{i \tau}^{w+}, \vec{a}_{i \tau}\left\{\left[W_{i-}^{*}\left(l_{k}(t)-L_{k}(t)\right]+\dot{\vec{L}}_{k}(t)\right\}^{-1}=\vec{a}_{i \tau}^{w-}\right.\right.
$$

while their initial projections $\vec{a}_{i \tau o}^{w}\left(\vec{a}_{i \tau o}^{w+}, \vec{a}_{i \tau o}^{w-}\right)$ follow from

$$
\alpha_{i \tau} \delta t \rightarrow \alpha_{i o} \delta \vec{l}_{k}=\vec{a}_{i \tau o}^{w-}+\vec{a}_{i \tau o}^{w-}
$$

Time-shift $\delta t$ depicts the observer processes with initial conditions $(4.2,4.3)$, which through inner controls $-2 \tilde{x}_{t \max }(\tau+o)=v^{+},-2 \tilde{x}_{t \max }(\tau+o)=v^{-}$start conjugated processes $(3.4 \mathrm{a}, \mathrm{b})$ with maxmin opposite speeds:

$$
\partial x_{i o \max } / \partial t=-2 \tilde{x}_{t o \max }(\tau+o) \vec{a}_{i \tau o}^{w+}, \partial x_{i o \min } / \partial t=-2 \tilde{x}_{t o \min }(\tau+o) \vec{a}_{i \tau o}^{w-} \text {, }
$$

where the projections of the initial conditions on rotating eigenvector $\vec{a}_{i \tau}^{w}$ determines the rotating projections of the process' initial speeds. The opposite speeds of the process (4.6), at the same fixed gradient $\partial x_{i} / \partial l_{k}$ (transforming the cutoff entropy), satisfy opposite rotations $\left(W_{i-}, W_{i+}\right)$ in $(3.4 \mathrm{a}, \mathrm{b})$ for each cutoff. Relation $\alpha_{i \tau} \delta t=u_{i \tau}$ sets an elementary entropy $u_{i \tau}$, generated at each cutoff by stepactions (4.1), which vector $\vec{a}_{i \tau}^{w}$ carries and transforms along the extremals (3.4a, b) according to (4.5, $4.5 \mathrm{a})$.

Each interval $\delta t$ determines the velocities by relations following from (4.5a),(4.6) at the known eigenvector $\vec{a}_{i \tau}$ and the eigenvalues, which are defined via diffusion or through rotating vector $\vec{a}_{i \tau}^{w}$ with entropy $u_{i \tau}$ and the above border eigenvalue. While the ratio of the eigenvector to its eigenvalue holds the space vector.

Hence, all parameters of the observer process are defined via the cutoff diffusion operator, and the observer covariant dynamics, emerge from the cutting fractions of Markov diffusion. The time-space movement illustrates Fig.1, with all analytical details in [10-14]. Conjugated processes (3.4a,b), starting within an impulse, head toward their entanglement, which minimizes the initially cut uncertainty that is transformed to initial conditions (4.2) of the certain dynamics. The invariant transformation leads to extreme process (at minimal information speed [13]):

$$
\partial x_{i} / \partial t+1 / 2 \sum_{k=1}^{3} \partial x_{i} / \partial l_{k} W_{i o}\left[l_{k}(t)-L_{k}(t)\right]=0
$$

with joint velocity $W_{i o}$ and the entangling dynamics minimizing entropy difference of processes (4.4a,b). For orthogonal rotations $W_{i+}[\vec{l}-\vec{L}]$ and $W_{i-}[\vec{l}-\vec{L}]$ at the entanglement, their modules hold relations 
International Journal o n Information Theory (IJIT ), Vol.3, No.4, October 2014

$\left|W_{i o}\right|=\sqrt{2}\left|W_{i \pm}[\vec{l}-\vec{L}]\right|_{\text {at }}\left|W_{i+}[\vec{l}-\vec{L}]\right|=\left|W_{i-}[\vec{l}-\vec{L}]\right|$.

The module of conjugated entangled orthogonal vectors $\vec{a}_{i \tau}^{w}=\left(\vec{a}_{i \tau}^{w+}, \vec{a}_{i \tau}^{w-}\right)$ at (4.7a) satisfies $\left|\vec{a}_{i \tau}^{w}\right|=\sqrt{2}\left|\vec{a}_{i \tau}^{w \pm}\right|$.

From $(4,7 \mathrm{a}, \mathrm{b})$ and (4.6) follow the connection of the modules for entangled speeds and states.

The ensemble's entropy pair $s_{a}^{i}, s_{b}^{i}$ holds complimentary of both conjugated pairs of rotating functions in $(3.4 \mathrm{a}, \mathrm{b})$ as an analogy of elementary wave functions that entangle the minimal cutoff entropy $s_{a b}^{i}=\mathrm{u}_{i}$, which is connected with the impulse initial entropy's instant $\mathrm{u}_{i o}$ at

$\mathrm{u}_{i}=\mathrm{u}_{i o} / \sqrt{2}$.

Additive probabilities of the complementary entropies provide additive complex amplitudes at entanglement, which satisfy symmetry condition. Verification of this condition reveals certain $a$ posteriori probability at entanglement. The elementary (quantum) dynamic process is prognosis at each time shift $\delta\left(t^{*}\right)$ of its random interactive action, generating uncertainty, which may convert the entangled entropy to equivalent information at $\delta\left(t^{*}\right) \rightarrow \delta(t), t \rightarrow t_{o}$.

The covariant dynamics, emerging from the cutting fractions of Markov diffusion, is a type of curved manifold known as a Riemannian symmetric space, where the space of diffusion tensor represents the covariance matrix in a Brownian motion. Since both step-functions are parts of the impulse entropy instant $\mathrm{u}_{i o}$, each of them caries entropy $\sim\left(\mathrm{u}_{i o}-\mathrm{u}_{i}\right) / 2$, and finally all impulse entropy is converting to equivalent information $\mathbf{a}_{i o} \cong \sqrt{2} \mathbf{a}_{i}$. Real control accumulates information $\left(\mathbf{a}_{i o}-\mathbf{a}_{i}\right)$ equivalent to twice of entropy for each virtual stepwise function, while the rising a posteriori probability increases entropy measure up to $\mathrm{u}_{i o} \rightarrow \mathbf{a}_{i o}$, at joining the impulse' step-wise functions. The max-min eventually limits both fractions along the trajectory.

Integration of these max-min portions of information along each observer's rotating conjugated trajectories, defined in four-dimensional space-time region $G_{4}^{*}$ with elementary volume $d v=d(\vec{l} \times t)$ as the integrant measure along trajectories, determines information path functional:

$I_{a p}=\int_{G_{4}^{*}, m \rightarrow \infty} d v \mathbf{a}_{i} \delta(\tau+t)\left(W_{i o}^{+}\right)^{-1}$,

where moment $\tau$ is a beginning of each real control connecting the process dimensions $m$.

Since $\mathrm{s}_{a p}^{i} \rightarrow \mathbf{a}_{i o}$ at each conversion, the $\mathrm{s}_{a p}^{i} \rightarrow S_{a p}$ extremals prognosis both the entropy dynamics of a virtual uncertain process within each interacting impulses, carrying uncertainty, and the information dynamics of the certain impulse, carrying information.

Each cutting action $u^{a}\left(\tau_{*}\right)$ and $\tilde{u}^{p}\left(\tau^{*}\right)$ potentially curtails the random process' fractions $\tilde{x}_{t}\left(\tau_{*}\right)$ and $\tilde{x}_{t}\left(\tau^{*}\right)$ on interval $\Delta_{*}$, killing the cutoff by moment $\tau_{2 k}$ (Fig.1) and converting entropy portion $\mathrm{s}_{a p}^{i}=\tilde{s}_{a p}^{o}\left[\tilde{x}_{t}\left(\Delta_{*}\right)\right] \rightarrow \tilde{s}_{a p}\left[\tilde{x}_{t}(\Delta)\right]$ to related information functional portion $i_{\delta}^{i}=i_{a p}^{o}\left[x_{t}\left(\Delta_{o}\right)\right]$ using the step-up control's $u^{p}\left(\tau_{*}\right)$ during the conversion process $x_{t}\left(\Delta_{o}\right), \Delta_{o}=\Delta_{1}+\Delta_{2}$, where the related symbols indicate random $\Delta$ and non-random $\Delta_{o}$ intervals. The conjugated dynamics proceed on interval $\Delta_{1}-\delta_{o}$, with interval $\delta_{o}$ of control $\tilde{u}^{p}\left(\tau^{*}\right)$ that switches from $\tau_{1 k}$ to $\tau_{1 k o}$. The entangled 
International Journal o n Information Theory (IJIT ), Vol.3, No.4, October 2014

entropy at $\tau_{1 k}$, is transformed during a gap $\delta_{o}$, and then at $\tau_{1 k o}$, the joint (inner) control $v_{t}$ unifies the information dynamics on interval $\Delta_{2}$ up to $\tau_{2 k}$-locality of turning it off.

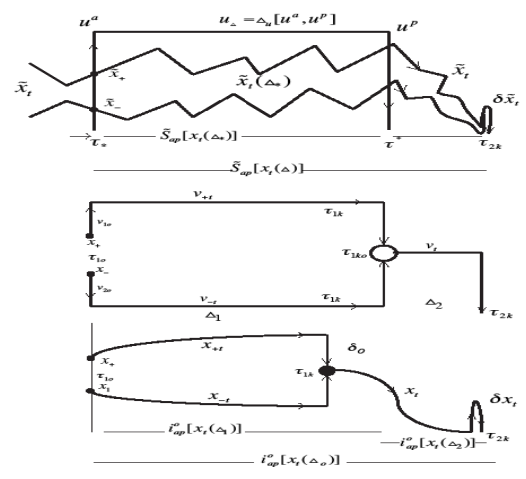

Figure1. Illustration of the observer external and internal processes and holding information.

Here: $\tilde{x}_{t}$ is external multiple random process, $\tilde{x}_{t}(\Delta)$ is potential observation on interval $\Delta$, which randomly divides $\tilde{x}_{t}$ on a priory $\tilde{x}_{a}(t)$ and a posteriori $\tilde{x}_{p}(t)$ parts; $u_{\Delta}=\Delta_{u}\left[u^{a}, u^{p}\right]$ are impulse control of parts $\tilde{x}_{a}(t), \tilde{x}_{p}(t) ; \tilde{s}_{a p}\left[\tilde{x}_{t}(\Delta)\right]$ is observer's portions of the entropy functional; $\tilde{x}_{t}\left(\Delta_{*}\right)$, $\Delta_{*}, u^{a}\left(\tau_{*}\right), \tilde{u}^{p}\left(\tau^{*}\right)$ and $\tilde{s}_{a p}\left[\tilde{x}_{t}\left(\Delta_{*}\right)\right]$ are related indications for each cutting process; $x_{t}\left(\Delta_{o}\right)$ is observer's internal process with its portion of information functional $i_{a p}^{o}\left[x_{t}\left(\Delta_{o}\right)\right] ; \tau_{2 k}$ is ending locality of $\tilde{x}_{t}$ with its sharp increase $\delta \tilde{x}_{t} ; \tilde{x}_{-}, \tilde{x}_{+}$are the cutting maximum information states; $v_{o}\left(v_{1 o}, v_{2 o}\right)$ are observer's opposite inner controls starting with $x_{-}\left(\tau_{1 o}\right), x_{+}\left(\tau_{1 o}\right)$ complex conjugated trajectories $x_{-t}, x_{+t}$ interfering nearby moment $\tau_{1 k} ; v_{+t}=f\left(x_{+}, x_{+t}\right), v_{-t}=f\left(x_{-}, x_{-t}\right)$ are inner control functions; interfering nearby moment $\tau_{1 k} ; \delta_{o}$ is interval of the control switch from $\tau_{1 k}$ to $\tau_{1 k o}$, where unified mirror control $v_{t}$ entangles the dynamics on interval $\Delta_{2}$ up to $\tau_{2 k}$ locality of turning the constraint off with sudden rise $\delta x_{t}$. The shown external and internal intervals could have different time scale.

On the way to entanglement, the controls minimizes the entropy speeds by imposing the VP constraint, which conserves the entropy carrying by the controls.

The controls' turn off frees this entropy as equivalent information $\mathbf{a}_{i o}-\mathbf{a}_{i}=\mathbf{a}_{i}(\sqrt{2}-1)$.

The added (free) information joins the rotating extremal segments.

Control $u^{a}\left(\tau_{*}\right)$ (Fig.1) converts portion $\tilde{s}_{a p}^{o}\left[\tilde{x}_{t}\left(\Delta_{*}\right)\right]$ to $i_{a p}^{o}\left[x_{t}\left(\Delta_{1}\right)\right]$ and concurrently starts the observer's process on interval $\Delta_{2}$, finishing it by the moment of killing $\tau^{*}+\Delta_{2} \rightarrow \tau_{2 k}$, where $\Delta_{*} \cong \tau_{1 k o}-\tau_{1 o}, \Delta_{1}=\tau_{1 k}-\tau_{1 o}+\delta_{o}, \delta_{o} \cong \tau_{1 k o}-\tau_{1 k}, \Delta \rightarrow \Delta_{o}$. 


\section{International Journal o n Information Theory (IJIT ), Vol.3, No.4, October 2014}

Killing Brownian motion can take a sharp increase at locality of hitting a time varying barrier [9],[14], (Fig.1), also resulting from the freeing of the control information.

The rotation, applied to each already ordered pairs of eigenvectors, equalizes their eigenvalues and binds them first in a double and then in a triple structure, which enfolds a minimal information, requested by the minimax.

Equal frequencies of complementary eigenvalues enable performing their cooperation through resonance[21].

Physical examples of emerging collective phenomena through active rotation are in [20].

While the impulse step-up action sets a transition process from the observing uncertainty to information, the impulse's certain step-down control cuts a minimum from each maxima of the observed information and initiates internal distributed information dynamics process, starting both the information and its observer (Fig.1).

The entanglement encloses the captured complimentary (conjugated) entropy fractions providing a source of an information unit.

The impulse step-up action launches the unit formation, while its certain control step-down action finishes the unit formation bringing memory and energy from the interactive jump decorreling the entangled entropy. This finite jump transits from uncertain Yes-logic to certain information No-logic, transferring the entangled entropy of observation to the forming information unit of elementary (quantum) bit. Uncertain step-up logic does not require energy [19] like the probes of observablevirtual process, or a Media whose information is not observed yet.

This potential (uncertain) logic belongs to sequential test-cuts before appearance of a priori certain logic, which becomes a part of forming the elementary information unit.

The entanglement might connect two (or three) distinguished units with superimposing ebits, whose measurement memorizes their information.

The Yes-No logic holds Bit-Participator in its forming as elementary information observer analogous to Wheeler Bit [6] created without any physical pre-law.

The curved control, binding two units in a doublet and cooperating its opposite directional information unit, forms a triplet as a minimal cooperative stable structure.

Resonance of the equal frequencies of complementary eigenvalues also performs their cooperation in doublet and triplet. Triplet structure can shape both naturally and artificially [21-23], when multiple interacting particles' units assemble collective observer with information process and building information forces [15].

\section{FORMING INFORMATION MACRODYMAMIC PROCESS}

The information path functional (IPF) integrates the multiple information units of cooperating doublets-triplets, bound by the free information, in gradual information flow of a physical macroprocess, which starts from statistical process whose multiple frequencies' test discloses the quantum microdynamics. The EF mathematical expectations average the random entropy impulses, and the controls covert them to microdynamics collected by the IPF, while transferring EF to IPF.

The IPF extremal trajectories describe the information macroprocess of the flows cooperating macrounits, which connects the averaged quantum microdynamics on the IPF extremals with classical Physical Statistical Physics and Thermodynamics in Observer.

The selective statistics of minimax probes, which minimize probes number, specify and distinct the considered observer macroprocess from that is not a selective observed. 


\section{International Journal o n Information Theory (IJIT ), Vol.3, No.4, October 2014}

This leads to three kinds of certain macroprocesses in observer: One that is formed according to minimax with sequential decreasing entropy and maximizing information enfolded in the sequence of enclosing triplet structures, while minimizing their bound free information.

This is a physical thermodynamic process generating the information network (IN) (Fig.2) with sequential information coupling (in doublets and their coupling in triplets) and rising information forces that enfold the ordered sequence and enable attracting new information unit by freeing the information.

The second kind brings an extreme to the path functional but does not support the sequential decrease, minimizing information speed by the end of forming the information unit. Such units might not be prepared to make optimal triple co-operations and generate the IN structures.

This is a physical macrodynamic process, which we classified as an objective or such one that is closed to a border threshold [15] with observer's subjective process, enables sequentially cooperate more than single triplet does in a process.

The third kind macroprocess, transforming energy-information with maximum of entropy production, which is destructive and does not support the observer stability, evolvement and evolution through the information network (IN), while, holding the elementary triplet, it forms the objective information observer.

The information Hamiltonian linear and nonlinear equations describe irreversible thermodynamics of all three kinds, which include the information forms of thermodynamics flows $\dot{x}=a^{u}$, defined via the IPF gradients as the process' information forces $X=(2 b)^{-1} a^{u}$ raised from the Markov process (Sec.1). The process Hamiltonian regularities arise at imposing dynamic constraint, initiated by the cutoff Markov diffusion; it blocks the initial randomness during intervals $\tau$ of applying the cutoff impulse [10]:

$a^{u}(\tau) X(\tau)+b(\tau) \frac{\partial X}{\partial x}(\tau)=0$.

The IN optimal co-operations hold observer's optimal macrostructures, but they are not necessary for a non-optimal sequence of units that forms a physical macrodynamic process, where each next unit randomly transfers energy-information to the following in various physical movements, including Markov diffusion.

These natural interactions routinely implement the conversion of pre-existence uncertainty to post interactive certainty through pre-interactive (a priori) and post-active (a posteriori) Bayesian probabilities.

Even if such multiple cooperation brings an extreme of the path functional, each following unit may not decrease the extremum, while each IN's triplet minimizes the bound information-energy that they enclosed, including triple quantum information.

Implementation of the variation principle requires compensation entropy production for the irreversibility by equivalent negentropy production, synchronized by Maxwell Demon[24].

On a primary quantum information level, it accomplishes the sequence of Yes-No, No-Yes actions following from the minimax at the cutoff [15]. A priori step-up Yes-control transfers total cutoff entropy (including the impulse's entropy) to equivalent information, and the posteriori step-down No-control kills the entropy while compensating it by equal information of the control.

Such control models an interaction coming from natural physical process like earthquake others, as observable process, being uncertain for potential observer. These natural interactions are a source, creating objective observer with the elementary doublets and triplets. The triplet, carrying naturally born free information, enables use it for cooperation with other naturally born doublets -triplets. If such cooperation brings information, allowing to overcome the threshold with subjective observer, it starts the subjective observer's IN with ability of free information to attract new triplets' information. 


\section{International Journal o n Information Theory (IJIT ), Vol.3, No.4, October 2014}

The end of Bayesian a priori interactive action kills the impulse entropy, while its time transition to a Bayesian posteriori inferring action delivers this entropy-information cost for converting that entropy to information bit, which is estimated by coefficient $k_{e}=s_{e v} / \ln 2 \cong 0.0917554$ [16].

Here, the real posteriori part of the impulse brings information equivalent, which covers entropy cost $s_{e v}$ and brings the real step-up control of a bordered next impulse. If an external step-down control, which is not a part of the impulse, spends the same entropy, and kills a cutting part of the impulse entropy $\mathrm{u}_{i}$, then $k_{e u}=s_{e v} / \mathrm{u}_{i} \approx 0.1272$.

Time interval of the conversion gap $\delta_{o}$ is $\delta_{t}^{o}=\mathrm{u}_{o i} / c_{o i}$ where a speed of killing this entropy is $c_{o i}$. Since by the end of $\delta_{o}$ the information unit appears with amount $\mathbf{a}_{i o}=\mathrm{u}_{o i}$, the real posteriori speed $c_{o i}$ produces the finite posteriori control speed.

Thus, this is speed of generating information, whose maximum estimates constant $c_{m i}=\hat{h}^{-1} \cong\left(0.536 \times 10^{-15}\right)^{-1}$ Nat / sec [13], where $\hat{h}$ is an information analog of Plank constant at maximal frequency of energy spectrum of information wave in its absolute temperature. This allows us to estimate minimal time interval $\delta_{t \text { min }}^{o} \cong \mathbf{a}_{i o} \hat{h} \approx 0.391143 \times 10^{-15} \mathrm{sec}$, which determines the ending border of generation information unit $\mathbf{a}_{i o}$. This unit contains an information equivalent $s_{e v}=i_{e v}$ of energy $e_{e v}$ spent on converting the entropy to information.

Energy $e_{e v}$ delivers the certain step-up control action of converting entropy to information.

This energy compensates for that conserved in the entangled rotation, specifically by angular moment multiplied on angular speed during the time movement [10], which evaluates entropy $s_{\text {evo }}$ in Nats. Above ratio $k_{e}$, measured by the equivalent cost of energy, is a quantum process' analogy of Boltzmann constant (as a ratio of the unit generating radiation energy, which is transferred to temperature of heat that dissipates this energy).

The ratio determines a part of total information $\mathrm{a}_{o i}$ cost for transforming entropy $\mathrm{u}_{o i}$. This information cost is a logical equivalent of Maxwell Demon's energy spent on this conversion, as the cost of logic, which is necessary to overcome $\delta_{o}$, including the transferring of the entangled entropy and generation of the unit of information during time interval $\delta_{t}^{o}$. Since the rotating movement condenses its entropy in the volume of entanglement, this logical cost is a primary result of time shift $\delta_{t}$ initiating the rotation, while this time course compensates for both logical and energy costs, delivering the certain step-wise control. This means, that real time course might be enough for excluding any other costs for conversion entropy in equivalent information.

Information mass [14] of rotating cooperating units, acquiring energy after entanglement, models elementary particles mass, as well as their various collective formations at the curving knots as the IN nodes (Fig.2),[25].

The step-up cut jumps the curvature of the space-time distribution, initiating an attractive wave of cooperation. A conjugated quantum process is reversible until it reaches entanglement at superposition (interaction) of the process' complimentary entropy fractions, directed toward the generation of information. The potential equalization uncertainty-certainty requires the existence of the extreme process, heading for both equalization and creation of the information unit with maximum information speed. The extreme condition [10],[11] compensates for entropy production through the VP Hamiltonian, measuring integral speed of the cooperating information process, which actually performs Maxwell's Demon function at macrolevel. The condition of the minimization of this speed (imposed by the VP dynamic constraint (5.1),[13]) limits information-energy for emerging unit by cutting it at formation and freeing the conserved information [16]. 
International Journal o n Information Theory (IJIT ), Vol.3, No.4, October 2014

The units of information had extracted from the observed random process through its minimax cutoff of hidden information which observer physical thermodynamic process integrates via Eigen Functional $[26,10]$, satisfying the VP on a macrolevel in the IPF form.

The IPF holds the generated primary free information that sequentially connects the pairs of statesunits as doublets and the multiple doublets-triplets co-operations.

\section{Arising Observer Logical Structure}

The triplet generates three symbols from three segments of information dynamics and one impulsecode from the control. This control joins all three in a single unit and transfers this triple to next triple, forming next level of the information network's (IN) minimal logical code. Each information unit has its unique position in the time-spaced information dynamics, which defines the scale of both timespace and the exact location of each triple code in the IN. Even though the code impulses are similar for each triplet, their time-space locations allows the discrimination of each code and its forming logics. The observer checks the acceptance of this code in IN (Fig.2).

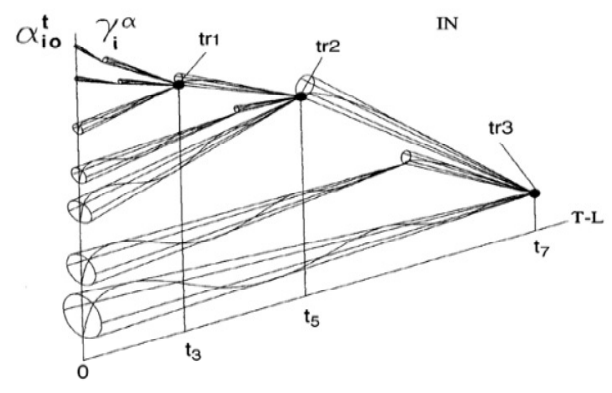

Figure 2. The IN time-space information structure.

The hierarchy of IN cones' spiral space-time dynamics with triplet nodes $(\operatorname{tr} 1, \operatorname{tr} 2, \operatorname{tr} 3, .$.$) , formed at$ localities of the triple cones vertexes' intersections with ranged string of the initial information speeds-eigenvalues $\alpha_{i o}^{t}$, cooperating around time locations $t_{1}, t_{2}, t_{3}$ in T-L time-space of the IN.

This includes enclosing the concurrent information in a temporary build IN's high-level logic that requests new information for the running observer's IN [15, v2].

If the code sequence satisfies the observer's IN code, the IN decreases its free information by enfolding new code in its structure. The IN information cooperative force, requests this compensating information [12]. The decrease is an indication that the requested information, needed for growing and extension of the IN logic has been received, which is associated with surprises that observer obtains from the requested information it needs.

The IN connections integrate each spatial position of the accepted triple code into the triplets' information of the IN node's previous position while structuring the IN. Timing of observer's internal spatial dynamics determines the scale of currently observed process and serves for both encoding and decoding the IN logic. The IN parameters are identified by observer's measured frequencies of the delivered information [15]. The spatial coordinate system rotates the following next accumulation and ordering of the IN node's information.

Thus, the dynamics generate the code's positions that define the logics, while the observer information creates both the dynamics and code. The space-time's position, following from the IN's cooperative capability, supports self-forming of observer's information structure (Fig.3), whose self-information has the IN distinctive quality measure. 
International Journal o n Information Theory (IJIT ), Vol.3, No.4, October 2014

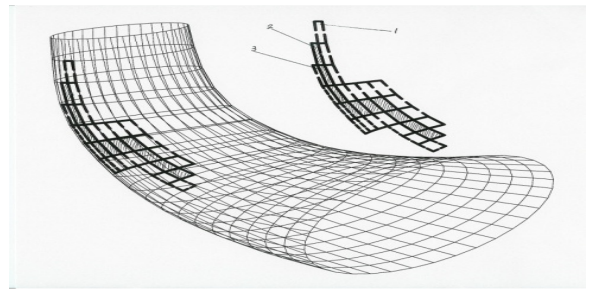

Figure 3. Illustration of the observer's self-forming cellular geometry by the cells of the triplet's code, with a portion of the surface cells (1-2-3).

The observer identifies order and measure of his priorities (like social, economic, others categories) using quality of information concentrated within the IN node, assigned to this priority [16].

The receiving information of this priority node's quality is directed to other (local) IN, which emanates from that node. This particular information is collected and enfolded according to the subpriority of competing variation's quantities of each category by analogy with main IN (Fig.2).

Each category of priority information builds its local IN emanating for the main IN. When the node quality is compensating by external information, the observer achieves this priority as unpredicted surprise; if it is not, the observer local IN requests for the new one.

The observer logic with its timing hierarchy initiates external interaction, following observation, entanglement, generation information units, and the IN nodes, forming its multiple finite sizes knots (Fig.2). This logic time interval could be minimizes for the sequential interactions approaching speed of light. In human observer's vision, the ability to see and form mental pictures has a speed much higher than external interactions [27].

Finally, the irreversible time course generates information with both objective and subjective observers, which can overcome the information threshold between them on the path to intelligence.

Observer computes its encoding information units in the information network (IN) code-logic to perform its integrating task through the self-generating program. The observing process, which is chosen by the observer's (0-1) probes and the logic integration, could determine such a program.

As a result, the information path functional (IPF) collects these information units, while the IN performs logical computing operations using the doublet-triplet code. Such operations, performed with the entangled memorized information units, model a quantum computation.

A classical computation models the operations with classical information units, which observer cooperates from quantum information units and runs in the IN with these units. An observer that unites logic of quantum micro- and macro- information processes enables composing quantum and/or classical computation on different IN levels. Observer kills uncertainty to get information.

\section{OBSERVER INTELLIGENCE}

The observer's current information cooperative force [15], initiated by its IN free information, determines the observer's selective actions and multiple choices, needed to implement the minimax self-directed strategy.

Notion of conscience $[28-29,16]$ we associate with selecting the optimal observer's choices through the cooperative force emanated from the integrated node. The selective actions are implemented via applying the considered stepwise controls with a feedback from the currently checked information density, requesting such spectrum information speed, which conveys the potential cooperation, needed to verify the chosen selection. 
International Journal o n Information Theory (IJIT ), Vol.3, No.4, October 2014

The cooperative force includes dynamic efforts implementing the observer's intention for attracting new high-quality information, which is satisfied only if such quality could be delivered by the frequency of the related observations through the selective mechanism [15].

These actions engage acceleration of the observer's information processing, coordinated with the highest density-frequency of observing information, quick memorizing each node information, and stepping up the generation of the encoded information with its logic and space-time structure, which minimizes the spending information. The time interval [15] of the control action predicts the needed selection, which is required to select the information that carries the requested information density.

These processes, integrating by the IPF, express the observer cognition, which starts with creation of the elementary information unit with the memories at microlevel.

The coordinated selection, involving verification, synchronization, and concentration of the observed information, necessary to build its logical structure of growing maximum of accumulated information, unites the observer's organized intelligence action, which the amount of quality of information spent on this action, being integrated in the IN node, currently evaluates.

The functional organization of the intelligent actions, which integrates cognition and conscience of the interacting observers, connects their levels of knowledge, evaluated by the hierarchical level's quality information [15] that the observers have accumulated and stored (memorized) in each IN.

The algorithm of multitasking intellectual network is in [30].

Results [31] confirm that cognition arises at quantum level as "a kind of entanglement in time"..." in process of measurement", where... "cognitive variables are represented in such a way that they don't really have values (only potentialities) until you measure them and memorize", even "without the need to invoke neurophysiologic variables", while "perfect knowledge of a cognitive variable at one point in time requires there to be some uncertainty about it at other times".

\section{CONCLUSION}

The identified Path from uncertainty to certainty integrates information quantum, classical, and computation processes in an observer intellect. The introduced integral path functional information measure implements the minimax law of optimal extraction and consumption of information for complex interactions. The results' computer-based methodology and software were applied to biological, intelligent, collective economic, social, and automatic control systems [10-16].

\section{REFERENCES}

[1] Bohr N. Atomic physics and human knowledge, Wiley, New York, 1958.

[2] Dirac P. A. M. The Principles of Quantum Mechanics, Oxford University Press (Clarendon), NewYork, 1947.

[3] Von Neumann J. Mathematical foundations of quantum theory, Princeton University Press, Princeton, 1955.

[4] Wheeler J. A. Quantum Mechanics, A Half Century Later Include the Observer in the Wave Function? Episteme 5:1-18, 1977.

[5] Wheeler J. A. On recognizing "law without law.” Am. J. Phys., 51(5), 398-404, 1983.

[6] Wheeler J. A. and Ford K. It from bit. In Geons, Black Holes \& Quantum Foam: A life in Physics, New York, Norton, 1998.

[7] Von Baeyer H.Ch. Quantum Weirdness? It's All In Your Mind. A new version of quantum theory sweeps away the bizarre paradoxes of the microscopic world. The cost? Quantum information exists only in your imagination, Scientific American, 4751, June 2013.

[8] Fuchs Ch.A. Quantum Bayesianism at the Perimeter,arXiv.org, 1003.5182, 2010.

[9] Ikeda N.,Watanabe S. Stochastic Differential Equations and Diffusion Process, College Press, University of Beijing, 1998. 


\section{International Journal o n Information Theory (IJIT ), Vol.3, No.4, October 2014}

[10] Lerner V.S. Information Path Functional and Informational Macrodynamics, Nova Science, New York, 2010.

[11] Lerner V.S. Solution to the variation problem for information path functional of a controlled random process functional, Journal of Mathematical Analysis and Applications,334:441-466, 2007.

[12] Lerner V.S. An observer's information dynamics: Acquisition of information and the origin of the cognitive dynamics, Journal Information Sciences, 184: 111-139, 2012.

[13] Lerner V.S. Hidden stochastic, quantum and dynamic information of Markov diffusion process and its evaluation by an entropy integral measure under the impulse control's actions, arXiv, 1207.3091, 2012.

[14] Lerner V.S. Hidden information and regularities of information dynamics I, 2,3, arXiv, 1207.5265, 1207.6563, 1208.3241, 2012.

[15] Lerner V.S. The information and its observer: external and internal information processes, information cooperation, and the origin of the observer intellect, arXiv, $1212.1710,2012$.

[16] Lerner V.S. Integrating hidden information which is observed and the observer information regularities, arXiv, 1303.0777, 2014.

[17] Kolmogorov A.N. Foundations of the Theory of Probability, Chelsea, New York, 1956.

[18] Kolmogorov A.N., Jurbenko I.G., Prochorov A.V. Introduction to the Theory of Probability, Nauka,1982.

[19] Bennett C.H. Logical Reversibility of Computation, IBM J. Res. Develop, 525-532. 1973.

[20] Nguyen H. P., Klotsa D. , Engel M., and Glotzer S.C. Emergent Collective Phenomena through Active Rotation, Physical Review Letters, 112, 075701, 2014.

[21] Efimov V.N.Weakly-bound states of three resonantly- interacting particles, Soviet Journal of Nuclear Physics, 12(5): 589-595,1971.

[22] Chakrabarty A., Konya A., Feng Wang F., Selinger J., V., and Wei Q. Brownian Motion of Boomerang Colloidal Particles, Physical Review Letters 111, 160603, 2013.

[23] Bologna M., West B. J,. and Grigolini P. Renewal and memory origin of anomalous diffusion, Physical Review Letters, E88,062106, 2013.

[24] Deffner S. Information driven current in a quantum Demon Maxwell, Physical Review Letters, E88, 062128, 2013.

[25] Kauffman L. H. Formal Knot Theory, Princeton Univ. Press, 1983.

[26] Feynman R.P. The character of physical law, Cox and Wyman LTD, London, 1963.

[27] Koch K., McLean J., Berry M., Sterling P., Balasubramanian V. and Freed M.A. Efficiency of Information Transmission by Retinal Ganglion Cells, Current Biology, 16(4), 1428-1434,2006.

[28] Heidelberger M. Nature from within: Gustav Theodor Fechner and his psychophysical worldview. Transl. C. Klohr, University of Pittsburg Press, USA, 2004.

[29] Pessoa L. and Engelmann J. B. Embedding reward signals into perception and cognition, Frontiers in Neuroscience, 4: 1-8, 2010.

[30] Sarkar A., and Mandal J. K. Computational Intelligence Based Simulated Annealing Guided Key Generation In Wireless Communication,International Journal. on Information Theory, 2(3),36-44, 2013.

[31] Yearsley J.M. and Pothos E. M. Challenging the classical notion of time in cognition: a quantum perspective, Proceedings of The Royal Society B, 3056.2013.

\section{AUTHOR}

Prof. Vladimir S. Lerner has taught at California University at Los Angeles, West Coast University, University, and in former USSR Universities (currently retired). He also lectures and consults worldwide. Dr. Lerner is the author and founder of Informational Macrodynamics- new computerbased Information Systems Science, author of 6 scientific books, more than 200 research articles, and 24 inventions. Professor Lerner biography is in "Who is who in America", "Who is who in the World".

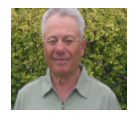

